\title{
USO DE MEDICAMENTOS COM AÇÃO ANTI-INFECCIOSA VIA SONDA GASTROENTERAL: RECOMENDAÇÕES PARA A ENFERMAGEM*
}

\author{
Moara Avila de Jesus Moreira ${ }^{1}$, Paula Pereira de Figueiredo ${ }^{2}$, Rosemary Silva da Silveira ${ }^{2}$, Liziani Iturriet Avila ${ }^{2}$, \\ Bárbara Tarouco da Silva ${ }^{2}$, Aline Martins Praxedes Silva ${ }^{3}$
}

RESUMO: Objetivo: apresentar recomendações específicas no preparo e administração de medicações antiinfecciosas via sonda gastroenteral no Serviço de Pronto Atendimento de um Hospital Universitário no Sul do Brasil. Método: estudo quali-quantitativo, descritivo-exploratório e de corte transversal, realizado entre abril e outubro de 2015. Quantitativamente utilizou-se a estatística descritiva e qualitativamente uma revisão não sistemática da literatura, em publicações nacionais e internacionais, a partir da combinação das palavras chave: perfil e medicações via sonda. Resultados: entre 568 prescrições analisadas, houve 143 medicamentos diferentes prescritos, com prevalência da classe de anti-infecciosos $(25,9 \%)$ e predominância das apresentações sólidas; contudo, 83,3\% dos medicamentos apresentavam alguma restrição de uso via sonda mesmo havendo opções de substituição disponíveis, demonstrando necessidade de protocolo assistencial. Considerações finais: entre a maioria das medicações sólidas prescritas via sonda gastroenteral, havia opções de substituição disponíveis, sendo necessária sua substituição por outras apresentações e o seguimento de recomendações específicas pela equipe de enfermagem/saúde.

DESCRITORES: Nutrição enteral; Segurança do paciente; Interações de medicamentos; Interações alimentodroga; Cuidados de enfermagem.

\section{USE OF ANTI-INFECTIVE MEDICATIONS VIA THE GASTROSTOMY CATHETER: RECOMMENDATIONS FOR NURSING}

ABSTRACT: Objective: to present specific recommendations in the preparation and administration of anti-infective medications via the gastrostomy catheter in the Emergency Unit of a University Hospital in the South of Brazil. Method: a descriptive-exploratory, cross-sectional, qualitative-quantitative study conducted between April and October 2015. Quantitative descriptive statistics were used, with a qualitative non-systematic review of the literature, considering national and international publications, from the combination of the keywords: profile and medications via catheter. Results: among the 568 prescriptions analyzed, there were 143 different medications prescribed, with prevalence of the anti-infectious class $(25.9 \%)$ and predominance of solid forms; however, $83.3 \%$ of the medications presented some restriction in use with catheter even though there were substitution options available, demonstrating the need for a care procedure. Final considerations: among the majority of the prescribed solid medications for use via gastrostomy catheter, replacement options were available, demonstrating the need for their substitution with other forms and for the follow-up of specific recommendations by the nursing/health team.

DESCRIPTORS: Enteral nutrition; Patient safety; Drug interactions; Food-drug interactions; Nursing care.

\section{USO DE MEDICAMENTOS CON ACCIÓN ANTIINFECCIOSA POR SONDA GASTROENTERAL: RECOMENDACIONES PARA LA ENFERMERÍA}

RESUMEN: Objetivo: presentar recomendaciones específicas acerca del preparo y de la administración de medicamentos antiinfecciosos por medio de sonda gastroenteral en el Servicio de emergencia de un Hospital Universitario en Sur de Brasil. Método: estudio cualitativo y cuantitativo, descriptivo exploratorio y de cohorte transversal, que se realizó entre abril y octubre de 2015. Para el análisis cuantitativo, se utilizó la estadística descriptiva y, para el análisis cualitativo, una revisión no sistemática de la literatura en publicaciones nacionales e internacionales, por medio de la combinación de las palabras clave: perfil y medicamentos por medio de sonda. Resultados: entre 568 prescripciones analizadas, hubo 143 medicamentos distintos prescriptos, con prevalencia de los antiinfecciosos $(25,9 \%$ ) y predominancia de las presentaciones sólidas; sin embargo, $83,3 \%$ de los medicamentos presentaban alguna restricción de uso por sonda mismo existiendo opciones de sustitutos disponibles, lo que muestra necesidad de protocolo asistencial. Consideraciones finales: entre la mayoría de los medicamentos sólidos prescriptos por sonda gastroenteral, había opciones de sustitución disponibles, siendo necesario sustituirlos por otras presentaciones además de obedecer a las recomendaciones específicas por el equipo de enfermería/salud.

DESCRIPTORES: Nutrición enteral; Seguridad del paciente; Interacciones de medicamentos; Interacciones alimento-droga; Cuidados de enfermería.

*Artigo extraído da dissertação "Medicações utilizadas por via oral e sonda gastroenteral em um Serviço de Pronto Atendimento: perfil e cuidados". Universidade Federal do Rio Grande, 2017.

${ }^{1}$ Enfermeira. Mestre em Enfermagem. Universidade Federal do Rio Grande. Rio Grande, RS, Brasil.

${ }^{2}$ Enfermeira. Doutora em Enfermagem. Docente de Enfermagem da Universidade Federal do Rio Grande. Rio Grande, RS, Brasil.

${ }^{3}$ Enfermeira. Universidade Federal do Rio Grande. Rio Grande, RS, Brasil.

Autor Correspondente:

Moara Avila de Jesus Moreira

Universidade Federal do Rio Grande

R. General Osório s/nº 96201-900 - Rio Grande, RS, Brasil

E-mail:moarajesus@yahoo.com.br
Recebido: 10/09/2017

Finalizado: 25/09/2018 


\section{- INTRODUÇÃO}

A administração de medicamentos é uma das atividades inerentes ao exercício da enfermagem. Para tanto, é preciso conhecimento teórico e prático, além de atenção redobrada na supervisão da equipe, já que o sistema de medicação inclui várias fases (prescrição, aprazamento, dispensação, preparo e administração), em que os erros acontecem com mais frequência do que o registrado ${ }^{(1-2)}$.

A via oral é a mais conveniente e, geralmente, a mais segura, com menor custo e, portanto, a mais comumente utilizada. No entanto, caso essa via esteja comprometida ou o paciente apresente dificuldade na deglutição, a sonda gastroenteral (SGE) pode ser uma alternativa na administração de medicamentos. Entretanto, a maioria dos medicamentos não é formulada para ser administrada por meio de uma sonda ${ }^{(3)}$.

De acordo com a Organização Mundial de Saúde (OMS), a (in)segurança do paciente é um grave problema de saúde pública mundial. Um em cada dez pacientes pode ser prejudicado ao receber cuidados em instituições de saúde. As causas dos erros são geralmente sistêmicas, tendo múltiplas origens como ambiente de trabalho desorganizado, falta de atenção e/ou concentração no preparo dos medicamentos, falta de informação sobre os medicamentos e o paciente ${ }^{(4)}$.

Especificamente sobre a administração de medicamentos via SGE, são poucos os estudos publicados por enfermeiros em âmbito nacional(5). Entretanto, os profissionais responsáveis pelo preparo e administração dos medicamentos precisam estar aptos para avaliar a forma farmacêutica a ser manipulada, a localização da sonda e possível interação da nutrição entérica (NE) com o fármaco. A obstrução da sonda está diretamente ligada à administração incorreta de medicamentos e dietas, em consequência de não haver irrigação após o uso ${ }^{(2-3,6)}$. A falta dessa prática desencadeia desconforto para o paciente e aumento da carga de trabalho para a equipe de saúde, podendo requerer a desobstrução da sonda ou mesmo a sua troca.

As formulações líquidas são a melhor escolha pela baixa probabilidade de obstrução da sonda. Contudo, não são todas as formas líquidas que podem ser administradas por essa via. É importante observar que os excipientes (como glicerol, propilenoglicol e sorbitol) e a alta osmolaridade de algumas preparações podem causar efeito adverso indesejado, aumentando o risco de intolerância do trato gastrintestinal. O grau de viscosidade, $\mathrm{pH}$ e tamanho das partículas em suspensão também precisam ser considerados para evitar a obstrução da sonda ${ }^{(7-8)}$.

Em quatro estudos realizados entre 2010 e 2012, verificou-se que a forma sólida foi referida como sendo maioria entre o perfil de medicamentos administrados por sonda em hospitais no Brasil ${ }^{(9-12)}$, caracterizando evidente predominância da indicação do prescrito por essa forma.

Todavia, ao preparar medicamentos na forma sólida para administração via SGE, é preciso conhecimento de como triturar, quando não triturar, com o que e qual o volume de líquido necessário para realizar a diluição. Como consequência da trituração, pode haver a aderência do medicamento triturado na parede da sonda e, também, ocorrer modificações na biodisponibilidade do princípio ativo do medicamento ${ }^{(3,9,13)}$.

Estudos $^{(7,14)}$ referem a dificuldade em obter informações técnicas dos fabricantes compatíveis com a literatura. Relatam a divergência de informações de outras fontes em $30 \%$ dos medicamentos analisados, tendo como referência o fabricante. Eles destacam ainda que o medicamento utilizado de forma diferente daquela prescrita pelo fabricante constitui uma prática offlabel, o que significa que o uso não é autorizado por uma agência reguladora; no entanto, isso não implica que seja incorreto.

Em um estudo ${ }^{(9)}$ verificou-se uma taxa significativa de erros no preparo de medicações via SGE, em formulações sólidas ou líquidas. A pesquisa considerou os aspectos de trituração, diluição e mistura de medicações. Uma vez que os fármacos não são manipulados com a finalidade para a qual foram desenvolvidos, o seu uso é baseado em empirismo ${ }^{(15)}$.

Pois isso, é relevante conhecer os medicamentos via oral e via SGE que são mais utilizados em unidades hospitalares, pois, a partir do levantamento preciso das medicações mais prescritas, é possível estabelecer protocolos que auxiliem no preparo e administração adequados para se manter 
a segurança do paciente. Assim, este estudo objetivou apresentar recomendações específicas para realizar o preparo e a administração de medicamentos via SGE do grupo de medicações anti-infecciosas em um Serviço de Pronto Atendimento (SPA) de um Hospital Universitário (HU) do Sul do Brasil.

\section{- MÉTODO}

Trata-se de um estudo quali-quantitativo, exploratório e descritivo, de corte transversal, desenvolvido no SPA de um HU no Sul do Brasil. O referido hospital possui atendimento exclusivo pelo Sistema Único de Saúde (SUS), sendo referência em diversas especialidades na região.

Esse estudo é parte de um macroprojeto de pesquisa, que buscou construir tecnologias de gestão e de cuidado aplicadas ao paciente adulto que faz uso de sonda como via para administração de medicamentos. Realizou-se, para tanto, um levantamento nas prescrições dos pacientes internados no SPA do referido HU entre de abril e outubro de 2015. Buscou-se, no mínimo, um medicamento prescrito por via oral e SGE, com aprazamento fixo. A coleta de dados ocorreu uma vez por semana, durante 27 semanas, em formulário próprio, no qual foram apreendidos os dados referentes à data de internação, nome do medicamento, forma de apresentação, dose, via e esquema de administração prescrita, considerando os pacientes internados naquele momento.

Construiu-se banco de dados no programa estatístico Statistical Package for Social Sciences (SPSS) versão 21.0, objetivando a análise descritiva da frequência dos medicamentos prescritos por via oral e por SGE mais utilizados nesse serviço ${ }^{(16)}$. Após análise inicial, os medicamentos identificados foram separados por grupo de ação, de acordo com o Manual de Padronização de Medicamentos $(2014)^{(17)}$ da instituição em estudo.

Elaborou-se a etapa qualitativa da pesquisa a partir da construção de uma lista com todos os medicamentos prescritos do grupo de ação com maior frequência no estudo: os fármacos de ação anti-infecciosa. Foram buscadas informações sobre alguma restrição na administração via SGE, bem como as particularidades da técnica de preparo e outras considerações relevantes associadas ao uso dessa via.

Além disso, foram sugeridas outras formas farmacêuticas alternativas para administração via SGE e outras vias quando disponíveis na instituição e no mercado. Tanto as restrições como as recomendações foram construídas por meio de uma revisão não sistemática da literatura, em publicações referentes ao tema, além de manuais de medicamentos de enfermagem e com base em consulta aos fabricantes, no sítio eletrônico da Agência Nacional de Vigilância Sanitária (ANVISA).

A busca bibliográfica foi considerada não sistematizada, pois houve dificuldade de obter estudos que apontassem recomendações específicas para o uso dos fármacos via SGE. Desse modo, partiu-se para uma procura ampliada na Biblioteca Virtual em Saúde (BVS), a partir da combinação das palavraschave: perfil e medicações via sonda. Essa busca foi desenvolvida no segundo semestre de 2016 e seus resultados incluíram artigos científicos, dissertações/teses e manuais de instituições como o Hospital Sírio Libanês (São Paulo/SP) e o Hospital Risoleta Tolentino Neves (Belo Horizonte/MG), considerandose o período de até cinco anos pregressos.

Esse estudo foi aprovado pelo Comitê de Ética em Pesquisa na Área da Saúde da Universidade a que está vinculado, sob parecer número 17/2015.

\section{- RESULTADOS}

O período em que ocorreu a coleta de dados obteve um total de 568 prescrições válidas para análise, nas quais se verificou a média de 8,5 dias de internação dos pacientes no SPA. Identificou-se que esse tempo de internação variou entre o mínimo de um dia e o máximo de 62 dias.

Inicialmente, foi realizado um perfil geral dos medicamentos prescritos via oral e por SGE no SPA, no qual foram identificados 143 medicamentos diferentes. A seguir, esses medicamentos foram classificados conforme o grupo de ação, de acordo com o Manual de Padronização de Medicamentos 
$(2014)^{(17)}$ da instituição pesquisada. A classe de medicamentos mais utilizada foi a de anti-infecciosos, com 37 (25,9\%) do total. A medicação Sulfametoxazol + Trimetoprima apresentou duas dosagens (400mg $+80 \mathrm{mg}$ e $800 \mathrm{mg}+160 \mathrm{mg}$ ), que se somaram no quantitativo final. Neste grupo de medicamentos, as apresentações sólidas predominaram e 83,3\% dos medicamentos apresentavam alguma restrição de uso via sonda, requerendo substituição por outras apresentações.

O Quadro 1 apresenta as medicações utilizadas segundo o grupo de ação anti-infecciosa verificadas no estudo, trazendo resultados referentes à forma farmacêutica prescrita na instituição, sua ação farmacológica e se há restrição via SGE ou não. Os fármacos em destaque são aqueles que tiveram as maiores frequências individuais.

Quadro 1 - Medicações de ação anti-infecciosa, prescritas por via oral e/ou por sonda gastroenteral no Serviço de Pronto Atendimento de um Hospital Universitário. Rio Grande, RS, Brasil, 2017 (continua)

\begin{tabular}{|c|c|c|c|c|}
\hline Nome do fármaco (dose) & $\begin{array}{c}\text { Nome } \\
\text { comercial / } \\
\text { Apresentação }\end{array}$ & $\begin{array}{l}\text { Forma farmacêutica } \\
\text { encontrada no estudo }\end{array}$ & $\begin{array}{c}\text { Ação } \\
\text { farmacológica }\end{array}$ & \begin{tabular}{|c} 
Administração \\
Via Sonda \\
S=SIM \\
N= NÃO \\
S*= SIM COM \\
RESTRIÇÕES
\end{tabular} \\
\hline Abacavir (300mg) & $\begin{array}{l}\text { Ziagenavir }{ }^{\circledast} \\
\text { ABC }\end{array}$ & Comprimido revestido & Antirretroviral & $\mathrm{S}^{*}$ \\
\hline Aciclovir (200mg) & Zovirax ${ }^{\circledast}$ & Comprimido simples & Antiviral & $\mathrm{S}$ \\
\hline Albendazol (400mg) & Zentel $^{\circledR}$ & Comprimido mastigável & Antiparasitário & $\mathrm{N}$ \\
\hline Amoxicilina (500mg) & Amoxil $^{\circledast}$ & Cápsula gelatina dura & Antibacteriano & $\mathrm{S}^{*}$ \\
\hline $\begin{array}{l}\text { Amoxicilina + Clavulanato } \\
\text { de Potássio }(500 \mathrm{mg}+125 \mathrm{mg})\end{array}$ & Clavulin $^{\circledast}$ & Comprimido revestido & Antibacteriano & $\mathrm{S}$ \\
\hline Ampicilina $(500 \mathrm{mg})$ & Binotal $^{\circledast}$ & Cápsula gelatina dura & Antibacteriano & $\mathrm{N}$ \\
\hline Azitromicina (500mg) & Zitromax $^{\circledast}$ & Comprimido revestido & Antibacteriano & $\mathrm{N}$ \\
\hline Ciprofloxacino (500mg) & Cipro $^{\circledast}$ & Comprimido simples & Antibacteriano & $\mathrm{S}^{*}$ \\
\hline Claritromicina $(500 \mathrm{mg})$ & Klaricid $^{\circledast}$ & Comprimido revestido & Antibacteriano & $\mathrm{N}$ \\
\hline Clindamicina (300mg) & Dalacin $^{\circledast}$ & Cápsula gelatina dura & Antibacteriano & $\mathrm{S}^{*}$ \\
\hline Dapsona & - & Comprimido simples & Antifúngico & $\mathrm{S}^{*}$ \\
\hline Efavirenz (600mg) & $\begin{array}{l}\text { Stocrin }^{\circledast} \\
\text { EFZ }\end{array}$ & Comprimido revestido & Antirretroviral & $\mathrm{N}$ \\
\hline Etambutol + Isoniazida* & - & Comprimido revestido & Tuberculostático & $\mathrm{N}$ \\
\hline Fluconazol (150mg) & Zoltec $^{\circledR}$ & Cápsula gelatina dura & Antifúngico & $\mathrm{S}^{*}$ \\
\hline Itraconazol (100mg) & Sporanox $^{\circledast}$ & Cápsula gelatina dura & Antifúngico & $\mathrm{N}$ \\
\hline Ivermectina (6mg) & Revectina $^{\circledR}$ & Comprimido simples & Antiparasitário & $\mathrm{N}$ \\
\hline Lamivudina (150mg) & $\begin{array}{l}\text { Epivir }^{\circledast} \\
\text { 3TC }\end{array}$ & Comprimido revestido & Antirretroviral & $\mathrm{S}$ \\
\hline $\begin{array}{l}\text { Lamivudina + Zidovudina } \\
(150 \mathrm{mg}+300 \mathrm{mg})\end{array}$ & Biovir $^{\otimes}$ & Comprimido revestido & Antirretroviral & $\mathrm{S}$ \\
\hline Levofloxacino (500mg) & Levaquin ${ }^{\circledast}$ & Comprimido revestido & Antibacteriano & $\mathrm{S}^{*}$ \\
\hline Lopinavir + Ritonavir & Kaletra $^{\circledR}$ & Comprimido revestido & Antirretroviral & $\mathrm{N}$ \\
\hline Metronidazol (400mg) & Flagyl $^{\circledast}$ & Comprimido revestido & \begin{tabular}{|c|} 
Antibacteriano e \\
antiparasitário \\
\end{tabular} & $\mathrm{N}$ \\
\hline Nitrofurantoína & Macrodantina ${ }^{\circledR}$ & Comprimido simples & Antibacteriano & $\mathrm{N}$ \\
\hline Ofloxacino $(600 \mathrm{mg})$ & Flogirax $^{\circledast}$ & Comprimido revestido & Antibacteriano & $\mathrm{N}$ \\
\hline Oseltamivir & Tamiflu $^{\circledR}$ & Cápsula gelatina dura & Antiviral & $\mathrm{S}^{*}$ \\
\hline Pirazinamida* (500mg) & - & Comprimido simples & Tuberculostático & $\mathrm{S}^{*}$ \\
\hline Pirimetamina (25mg) & Daraprim $^{\circledast}$ & Comprimido simples & Antiparasitário & $\mathrm{S}$ \\
\hline
\end{tabular}


Cogitare Enferm. (23)4: e55166, 2018

\begin{tabular}{|c|c|c|c|c|}
\hline Primaquina $(15 \mathrm{mg})$ & Primakinder $^{\circledast}$ & Drágea & Antiparasitário & $\mathrm{N}$ \\
\hline Raltegravir (400mg) & Isentress $^{\circledR}$ & Comprimido simples & Antirretroviral & $\mathrm{N}$ \\
\hline Rifabutina (150mg) & Mycobutin $^{\circledast}$ & Comprimido simples & $\begin{array}{l}\text { Antibacteriano e } \\
\text { tuberculostático }\end{array}$ & $\mathrm{S}^{*}$ \\
\hline $\begin{array}{l}\text { Rifampicina + Isoniazida + } \\
\text { Pirazinamida + Etambutol }\end{array}$ & $\mathrm{RHZE}^{\circledast}$ & Comprimido revestido & Tuberculostático & $\mathrm{N}$ \\
\hline Ritonavir (100mg) & $\begin{array}{l}\text { Norvir }^{\oplus} \\
\text { RTV }\end{array}$ & Cápsula gelatina mole & Antirretroviral & $\mathrm{N}$ \\
\hline Sulfadiazina (500mg) & Suladrin $^{\circledast}$ & Comprimido simples & $\begin{array}{c}\text { Antibacteriano e } \\
\text { antifúngico }\end{array}$ & $\mathrm{N}$ \\
\hline $\begin{array}{l}\text { Sulfametoxazol + } \\
\text { Trimetoprima - }(400 \mathrm{mg}+ \\
80 \mathrm{mg} \text { e } 800 \mathrm{mg}+160 \mathrm{mg})\end{array}$ & Bactrim $^{\circledast}$ & Comprimido simples & $\begin{array}{l}\text { Antibacteriano e } \\
\text { antifúngico }\end{array}$ & $\mathrm{N}$ \\
\hline Telzir & Fozamprenavir $^{\circledast}$ & Comprimido revestido & Antirretroviral & $\mathrm{N}$ \\
\hline Tenofovir & $\begin{array}{c}\text { Vired }^{\circledR} \\
\text { TDF } \\
\end{array}$ & Comprimido simples & Antirretroviral & $\mathrm{N}$ \\
\hline Tenofovir + Lamivudina* & - & Comprimido revestido & Antirretroviral & $\mathrm{N}$ \\
\hline
\end{tabular}

*Fármacos não comercializados, estando disponíveis somente nas unidades sanitárias dos serviços de saúde pública².

Nesse grupo, os comprimidos revestidos representaram 15 (40,6\%) apresentações, seguidos de 13 comprimidos simples $(35,1 \%)$, seis cápsulas de gelatina dura $(16,2 \%)$, uma cápsula de gelatina mole $(2,7 \%)$, uma drágea $(2,7 \%)$ e um comprimido mastigável (2,7\%). Dessas medicações, 23 (62,2\%) têm outra opção de substituição para administração, como suspensão oral ou solução injetável.

Dentre todas as medicações prescritas neste grupo, 22 (59,4\%) não podem ser administradas por SGE e 10 (27\%) podem, mas com algum tipo de restrição, apontando a necessidade urgente de um protocolo assistencial acerca dessa temática.

\section{DISCUSSÃO}

Sabe-se que a via de administração a ser utilizada depende das formas de apresentação dos medicamentos disponíveis e da condição clínica do paciente. Contudo, nesse e em outros estudos $^{(7,10-12,14-15,18)}$, constatou-se grande representatividade de apresentações sólidas, as quais têm possibilidade de troca para apresentações líquidas via gastroenteral ou mesmo endovenosa. Considerando a segurança do paciente em caso de uso via sonda, deve-se dar preferência para as apresentações líquidas, como suspensões, xaropes e soluções ${ }^{(8)}$.

O grupo de fármacos anti-infecciosos configura o conjunto de medicamentos e fórmulas responsáveis por combater bactérias, vírus, parasitas e fungos. Constituem a segunda classe medicamentosa com maior impacto financeiro para as instituições de saúde ${ }^{(19)}$. Dos medicamentos encontrados, destacaram-se as classes de antibacterianos, antiparasitários, antifúngicos, antivirais, antissépticos e os escabicidas e pediculocidas. O emprego de variados subgrupos de anti-infecciosos justifica-se pela alta complexidade dos casos clínicos que são atendidos no SPA e pelo longo período de permanência dos pacientes naquela unidade. Nesse grupo, Claritromicina, Sulfametoxazol + Trimetoprima, Pirimetamina e Azitromicina foram os fármacos com maior frequência de prescrição.

O medicamento Albendazol foi prescrito na forma de apresentação mastigável, mas também está disponível no mercado sob a forma de comprimido simples e de suspensão oral. O fabricante não relata como deve ser o preparo em caso de administração via SGE, apenas que os comprimidos devem ser mastigados ou tomados com água ${ }^{(20)}$. Entretanto, um autor refere em seu manual que os comprimidos bucais são concebidos para serem absorvidos pela mucosa oral e ultrapassar os efeitos do metabolismo de primeira passagem do fígado ${ }^{(21)}$, o que o contraindicaria o uso via sonda. 
ativo e os excipientes podem causar obstrução da sonda. É de fácil substituição por estar disponível na instituição em forma de suspensão oral e injetável ${ }^{(2,7,20,22)}$.

As cápsulas de Fluconazol e Amoxacilina devem ser abertas e seus componentes misturados em água para completa solubilização e, então, administradas via $\mathrm{SGE}^{(2,18,22-23)}$. Porém, indica-se buscar alternativa de substituição, pois há o risco da perda do conteúdo da cápsula ocasionando subdosagem ${ }^{(21)}$. Os fabricantes não referem outra forma de uso senão a via oral, na qual as cápsulas devem ser ingeridas inteiras ${ }^{(20)}$. Alguns autores ${ }^{(18,22)}$ referem não haver estudos sobre eficácia, segurança e farmacocinética, e não orientam a administração via SGE, sugerindo a substituição pela suspensão oral (Amoxacilina) ou injetável (Fluconazol).

Já os grânulos da cápsula de Itraconazol não se dissolvem em água, o que a impede de ser administrada via SGE. Se administrado via oral, deve ser imediatamente após uma refeição para obtenção do grau máximo de absorção. Pela ausência de outra alternativa de substituição, sugere-se a troca do medicamento por outro, de princípio ativo semelhante ${ }^{(20-22)}$. Para o medicamento Amoxacilina + Ácido Clavulânico, também é indicado o uso da suspensão oral e o fabricante não menciona os cuidados em caso de administração via SGE ${ }^{(20-21)}$.

Nos casos da Azitromicina, Metronidazol e da Sulfadiazina, os fabricantes apenas referem a administração por via oral, conforme indicação na bula ${ }^{(19)}$, e não recomendam que esses medicamentos sejam mastigados. Isso significa que os mesmos não deveriam sofrer alteração em sua forma de apresentação. Alguns autores ${ }^{(2,18,22,24)}$ orientam a substituição dos comprimidos de Azitromicina e Metronidazol por suspensão oral ou injetável, pois se triturados esses comprimidos podem levar à obstrução da sonda. Se a escolha for suspensão oral, esta necessita ser administrada uma hora antes ou duas horas após a NE, pois há redução significante da disponibilidade e eficácia quando administrado simultaneamente ${ }^{(18,22)}$.

Outros fármacos que podem apresentar interação com a NE são Ciprofloxacino, Clindamicina, Levofloxacino, Dapsona, Oseltamivir e Rifabutina. Eles podem ser administrados via SGE, porém, devem ser separados da NE, pois ela pode acarretar diminuição da absorção. Recomenda-se pausar a NE uma hora antes e reiniciá-la uma hora depois da administração dos fármacos ${ }^{(14-15,18,21-22)}$. Outros autores $^{(2)}$ acrescentam ainda que, no caso do Ciprofloxacino, as sondas gástricas produzem uma perda maior na absorção se comparadas às enterais. A solução injetável é alternativa de substituição para as três primeiras medicações.

A literatura desaconselha que os medicamentos Claritromicina, Ampicilina, Ivermectina, Nitrofurantoína, Ofloxacino e Primaquina sejam administrados via SGE, pois não apresentam estudos sobre eficácia, segurança e farmacocinética. Deve-se avaliar uso alternativo de Claritromicina e Ampicilina como suspensão oral e injetável, e da Nitrofurantoína na forma de suspensão oral(2,18,20-22). Os medicamentos combinados Etambutol + Isoniazida e Rifampicina + Isoniazida + Pirazinamida + Etambutol $\left(\mathrm{RHZE}^{\oplus}\right)$ também não apresentam dados de administração via $\mathrm{SGE}$, mas se usados via oral devem ser administrados, preferencialmente, em jejum ${ }^{(2,6,20)}$.

Empiricamente, no cotidiano da assistência de enfermagem, tem-se observado que esse medicamento forma uma pasta consistente quando triturado e diluído em água destilada, sendo necessária a utilização de água morna para melhor solubilizá-lo. Portanto, acredita-se que o risco de obstrução da sonda seja maior, assim como aumentam as chances de haver subdosagem, já que é mais difícil a diluição completa do medicamento. Além dessas, a Pirazinamida, que também foi prescrita individualmente, pode ser administrada via SGE, preferencialmente a nível gástrico e separada da NE. Como há o risco de obstrução do cateter, sugere-se a substituição pela solução oral ${ }^{(2,23)}$.

Aos fármacos de apresentação sólida com liberação imediata que não possuam alternativa de substituição no mercado ou na instituição, é recomendada a trituração até se obter um pó fino, que será dissolvido em 15 a $30 \mathrm{ml}$ de água destilada. Contudo, deve-se dar atenção aos comprimidos de liberação controlada, pois a trituração altera seu perfil de liberação, afetando significativamente seu efeito farmacológico. Além disso, a sonda deve ser lavada antes e após cada administração, a fim de evitar obstrução(8).

O uso de antirretrovirais (ARV) tem como objetivo melhorar a qualidade de vida do indivíduo, impedir a replicação do vírus e minimizar a mortalidade entre os infectados ${ }^{(19)}$. Dessa forma, para alcançar o 
objetivo terapêutico desejado, os fármacos não podem sofrer alterações em sua composição.

Cada classe de medicamento ataca o vírus HIV de uma maneira diferente. Geralmente combinamse medicamentos de duas ou até três classes para garantir ataque potente contra o vírus. A primeira classe de medicamentos (inibidores nucleosídeos da transcriptase reversa) é indicada como de primeira linha no tratamento da infecção pelo vírus HIV. O fármaco Abacavir (ABC) é utilizado em combinação com outros ARV. É encontrado na forma de apresentação de comprimido revestido, que, se administrado via oral, pode ser ingerido com ou sem alimentos. Mas, em caso de administração por sonda, o fabricante não menciona as especificidades de preparo e administração. Tem como alternativa de substituição a solução oral, que deve ser separada da NE. Salienta-se o cuidado com possíveis reações de hipersensibilidade, como sintomas sistêmicos respiratórios e/ou gastrintestinais, em geral com febre. A solução oral contém sacarina e sorbitol na composição, que pode causar dor abdominal e diarreia ${ }^{(2,19-20)}$.

Lamivudina + Zidovudina é uma combinação em dose fixa que aumenta a conveniência para o paciente. Foi prescrito na forma de apresentação de comprimido revestido, e, por ser combinado, esse medicamento não apresenta substituição, somente na forma separada, em que a Lamivudina (3TC) está disponível como solução oral e a Zidovudina (AZT) solução oral e injetável. O fabricante refere não haver problema na maceração dos comprimidos citados ${ }^{(20)}$. A Lamivudina (3TC), que também foi prescrita individualmente, é indicada para prevenção de transmissão materno-fetal de HIV e no tratamento de hepatite B crônica ${ }^{(19)}$.

Fechando a primeira classe de ARV, o Tenofovir (TDF) foi prescrito na sua forma individual e combinado Tenofovir+Lamivudina, na forma de apresentação de comprimido revestido. Este fármaco não tem outra opção de substituição disponível, e o fabricante contraindica a trituração do comprimido ${ }^{(20)}$.

O Efavirenz (EFZ) faz parte da segunda classe de ARV (inibidores não nucleosídeos da transcriptase reversa) e é utilizado em combinação com outros ARV. Deve ser administrado preferencialmente à noite e com o estômago vazio. Tem como alternativa de substituição a solução oral, já que o comprimido é de difícil trituração e o revestimento pode obstruir o cateter ${ }^{(2,19,21)}$.

Fozamprenavir é integrante da terceira classe de medicamentos ARV (inibidores da protease) e é um dos medicamentos de escolha em início de tratamento. O fabricante refere que o comprimido é destinado apenas à administração via oral; no entanto, tem a suspensão oral como alternativa de substituição. Não há estudos relacionados à farmacocinética na administração via SGE, mas caso necessário, orienta-se a preferência pela formulação líquida, separadamente da $\mathrm{NE}^{(20)}$.

O Lopinavir + Ritonavir foi prescrito como comprimido revestido, e o fabricante apenas refere a administração por via oral e indica a solução oral como alternativa de substituição. Diferente de outros medicamentos combinados que tem a possibilidade de administração separadamente, o Lopinavir está disponível somente em combinação com o Ritonavir. É importante destacar que essa combinação apresenta inúmeras e significativas interações ${ }^{(2,20)}$. Já o Ritonavir (RTV), separadamente, foi prescrito na forma de cápsula de gelatina mole e possui a solução oral como alternativa para administração via SGE, pois o fabricante orienta que o medicamento não pode ser partido, aberto ou mastigado ${ }^{(2)}$. Por fim, o Raltegravir, da quarta classe (inibidores da integrase), foi prescrito como comprimido simples; contudo, não há dados disponíveis que indiquem a administração via SGE ${ }^{(2)}$. O Quadro 2 apresenta uma síntese das orientações para administração dos fármacos via sonda e as alternativas de substituição disponíveis no mercado.

Quadro 2 - Síntese das orientações para administração dos fármacos de ação anti-infecciosa, via sonda e as alternativas de substituição disponíveis no mercado. Rio Grande, RS, Brasil, 2017 (continua)

\begin{tabular}{|l|l|l|}
\hline Nome do fármaco & Orientação para administração VS & Alternativa de substituição \\
\hline Abacavir & $\begin{array}{l}\text { Fabricante não menciona especificidades do preparo e } \\
\text { administração }{ }^{(2,19-20)} .\end{array}$ & $\begin{array}{l}\text { Solução oral, que deve ser } \\
\text { separada da NE. }\end{array}$ \\
\hline Albendazol & $\begin{array}{l}\text { Fabricante não menciona especificidades do preparo e } \\
\text { administração }\end{array}$ & $\begin{array}{l}\text { Comprimido simples e suspensão } \\
\text { oral. }\end{array}$ \\
\hline
\end{tabular}




\begin{tabular}{|c|c|c|}
\hline Amoxicilina & $\begin{array}{l}\text { As cápsulas devem ser abertas e seus componentes } \\
\text { misturados em água para completa solubilização }{ }^{(2,18,22-23)} \text {. }\end{array}$ & Suspensão oral. \\
\hline Ampicilina & $\begin{array}{l}\text { Não há estudos sobre eficácia, segurança e } \\
\text { farmacocinética }{ }^{(2,18,20-22)} \text {. }\end{array}$ & Suspensão oral e injetável. \\
\hline Azitromicina & $\begin{array}{l}\text { Fabricante não recomenda que estes medicamentos } \\
\text { sofram alteração em sua forma de apresentação } \mathrm{O}^{(20)} \text {. }\end{array}$ & $\begin{array}{l}\text { Suspensão oral (uma hora antes } \\
\text { ou duas horas após a NE) e } \\
\text { injetável(17,21). }\end{array}$ \\
\hline Ciprofloxacino & $\begin{array}{l}\text { Podem ser administrados via SGE, porém separados } \\
\text { da NE, pois ela pode acarretar diminuição da } \\
\text { absorção } \mathrm{O}^{(14-15,18,21-22)} \text {. }\end{array}$ & Solução Injetável. \\
\hline Claritromicina & $\begin{array}{l}\text { Não há estudos sobre eficácia, segurança e } \\
\text { farmacocinética }{ }^{(2,18,20-22)} \text {. }\end{array}$ & Suspensão oral e injetável. \\
\hline Clindamicina & $\begin{array}{l}\text { Podem ser administrados via SGE, porém separados } \\
\text { da NE, pois ela pode acarretar diminuição da } \\
\text { absorção } \mathrm{O}^{(14-15,18,21-22)} \text {. }\end{array}$ & Solução Injetável. \\
\hline Dapsona & $\begin{array}{l}\text { Podem ser administrados via SGE, porém separados } \\
\text { da NE, pois ela pode acarretar diminuição da } \\
\text { absorção }{ }^{(14-15,18,21-22)} \text {. }\end{array}$ & Não há. \\
\hline Efavirenz & $\begin{array}{l}\text { Sugere-se substituição, já que o comprimido é de } \\
\text { difícil trituração e o revestimento pode obstruir o } \\
\text { cateter }{ }^{(2,19,21)} \text {. }\end{array}$ & \begin{tabular}{|l} 
Solução oral. \\
\end{tabular} \\
\hline $\begin{array}{l}\text { Etambutol + } \\
\text { Isoniazida }\end{array}$ & $\begin{array}{l}\text { Fabricante não menciona especificidades do preparo e } \\
\text { administração via } \mathrm{SGE}^{(2,6,20)} \text {. }\end{array}$ & Não há. \\
\hline Fluconazol & $\begin{array}{l}\text { As cápsulas devem ser abertas e seus componentes } \\
\text { misturados em água para completa solubilização(2,18,22-23). }\end{array}$ & Injetável. \\
\hline Itraconazol & $\begin{array}{l}\text { Os grânulos das cápsulas não se dissolvem em água, o } \\
\text { que impede a administração via SGE }\end{array}$ & $\begin{array}{l}\text { Pela ausência de alternativa de } \\
\text { substituição, sugere-se a troca } \\
\text { do medicamento por outro, de } \\
\text { princípio ativo semelhante }{ }^{(19-21)} \text {. } \\
\end{array}$ \\
\hline Ivermectina & $\begin{array}{l}\text { Não há estudos sobre eficácia, segurança e } \\
\text { farmacocinética }{ }^{(2,18,20-22)} \text {. }\end{array}$ & \begin{tabular}{|l|} 
Suspensão oral e injetável. \\
\end{tabular} \\
\hline Levofloxacino & $\begin{array}{l}\text { Podem ser administrados via SGE, porém separados } \\
\text { da NE, pois ela pode acarretar diminuição da } \\
\text { absorção } \mathrm{O}^{(14-15,18,20-22)}\end{array}$ & \begin{tabular}{|l} 
Solução Injetável. \\
\end{tabular} \\
\hline $\begin{array}{l}\text { Lopinavir + } \\
\text { Ritonavir }\end{array}$ & $\begin{array}{l}\text { Fabricante não menciona especificidades do preparo } \\
\text { e administração. É importante destacar que essa } \\
\text { combinação apresenta inúmeras e significativas } \\
\text { interações }^{(2,20)} \text {. }\end{array}$ & Solução oral. \\
\hline Metronidazol & $\begin{array}{l}\text { Fabricante não recomenda que estes medicamentos } \\
\text { sofram alteração em sua forma de apresentação }{ }^{(20)} \text {. }\end{array}$ & $\begin{array}{l}\text { Suspensão oral (uma hora antes } \\
\text { ou duas horas após a NE) e } \\
\text { injetável }{ }^{(17,21)} \text {. }\end{array}$ \\
\hline Nitrofurantoína & $\begin{array}{l}\text { Não há estudos sobre eficácia, segurança e } \\
\text { farmacocinética }{ }^{(2,18,20-22)} \text {. }\end{array}$ & \begin{tabular}{|l|} 
Suspensão oral e injetável. \\
\end{tabular} \\
\hline Ofloxacino & $\begin{array}{l}\text { Não há estudos sobre eficácia, segurança e } \\
\text { farmacocinética }{ }^{(2,18,20-22)} \text {. }\end{array}$ & Suspensão oral e injetável. \\
\hline Oseltamivir & $\begin{array}{l}\text { Podem ser administrados via SGE, porém separados } \\
\text { da NE, pois ela pode acarretar diminuição da } \\
\text { absorção }{ }^{(14-15,18,20-22)} \text {. }\end{array}$ & Não há. \\
\hline Pirazinamida & Preferencialmente a nível gástrico e separada da $\mathrm{NE}^{(2,23)}$. & Solução oral. \\
\hline Primaquina & $\begin{array}{l}\text { Não há estudos sobre eficácia, segurança e } \\
\text { farmacocinética }{ }^{(2,18,20-22)} \text {. }\end{array}$ & Suspensão oral e injetável. \\
\hline Raltetravir & $\begin{array}{l}\text { Não há dados disponíveis que indiquem a administração } \\
\text { via SGE(2). }\end{array}$ & Não há. \\
\hline Rifabutina & $\begin{array}{l}\text { Podem ser administrados via SGE, porém separados } \\
\text { da NE, pois ela pode acarretar diminuição da } \\
\text { absorção } \\
{ }^{(14-15,18,20-22)} \text {. }\end{array}$ & Não há. \\
\hline
\end{tabular}




\begin{tabular}{|l|l|l|}
\hline $\begin{array}{l}\text { Rifampicina }+ \\
\text { Isoniazida }+ \\
\text { Pirazinamida }+ \\
\text { Etambutol }\end{array}$ & $\begin{array}{l}\text { Fabricante não menciona especificidades do preparo e } \\
\text { administração via SGE }\end{array}$ & Não há. \\
\hline Ritonavir & $\begin{array}{l}\text { Fabricante não recomenda que este medicamento } \\
\text { sofra alteração em sua forma de apresentação } 0^{(2)}\end{array}$ & Solução oral. \\
\hline Sulfadiazina & $\begin{array}{l}\text { Fabricante não recomenda que estes medicamentos } \\
\text { sofram alteração em sua forma de apresentação }\end{array}$ & Não há. \\
\hline $\begin{array}{l}\text { Sulfametoxazol } \\
+ \text { Trimetoprima }\end{array}$ & $\begin{array}{l}\text { Não deve ser triturado, pois o princípio ativo e os } \\
\text { excipientes podem causar obstrução da sonda }{ }^{(2,7,20,22)} .\end{array}$ & Suspensão oral e injetável. \\
\hline Telzir & $\begin{array}{l}\text { Não há estudos relacionados à farmacocinética, } \\
\text { mas caso necessário, orienta-se a preferência pela } \\
\text { formulação líquida, separadamente da NE } E^{(20)} .\end{array}$ & Suspensão oral. \\
\hline Tenofovir & Fabricante contraindica a trituração do comprimido ${ }^{(20)}$. & Não há. \\
\hline $\begin{array}{l}\text { Tenofovir }+ \\
\text { Lamivudina }\end{array}$ & Fabricante contraindica a trituração do comprimido & Não há. \\
\hline
\end{tabular}

FONTE: O autor (2017).

Não há na literatura uma fonte única que seja completa e que forneça recomendações específicas via sonda de todos os medicamentos. Somente os periódicos científicos, com pesquisas de foco principal nesse tema, apresentam algumas orientações. A divergência entre autores mostrou-se como dificuldade nesse e em outros estudos ${ }^{(7,25)}$, nos quais $30,4 \%$ e $39,5 \%$, respectivamente, dos medicamentos apresentaram divergência de informações em relação a outras fontes.

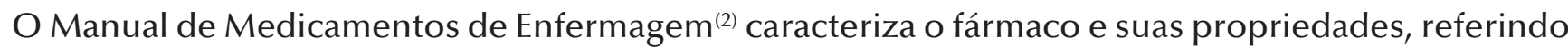
o uso via oral ou SGE; porém, não apresenta especificidades caso somente as apresentações sólidas estejam disponíveis. Já os fabricantes, que seriam a orientação de referência, não reconhecem a possibilidade de adaptação da forma oral para a via SGE, dificultando a correta direção a ser seguida, o que acaba responsabilizando os profissionais envolvidos por qualquer consequência que venha a ocorrer com pacientes sob este uso.

Além dos fabricantes, cabe destacar o compromisso do Ministério da Saúde e da ANVISA em estimular, regular e fiscalizar a constituição de formas farmacêuticas alternativas para os medicamentos anti-infecciosos, em especial os ARV e tuberculostáticos, já que muitos pacientes com as respectivas patologias são debilitados e dependem da sonda para receberem seu tratamento medicamentoso.

\section{CONSIDERAÇÕES FINAIS}

Os resultados encontrados nesse estudo evidenciaram medicações sólidas prescritas para serem administradas via SGE quando a via oral se torna inadequada. Contudo, para a maioria dos medicamentos, havia opções de substituição disponíveis.

Como limitações do estudo, considera-se a carência de pesquisas na temática da administração de medicamentos sólidos por SGE, assim como a falta de informações na própria bula dos fabricantes. Existe ainda divergência de informações entre os artigos e manuais, quando encontrados. Portanto, este trabalho constitui uma ferramenta importante no auxílio à equipe de saúde na escolha adequada para a via SGE e pode contribuir na segurança relacionada ao preparo e administração de medicamentos por esta via.

Sugere-se a realização de capacitações permanentes aos profissionais e pesquisas futuras em outros grupos de medicamentos com fármacos mais utilizados, igualmente importantes nas unidades hospitalares.

\section{REFERÊNCIAS}


1. Bohomol E. Erros de medicação: estudo descritivo das classes dos medicamentos e medicamentos de alta vigilância. Esc. Anna Nery. [Internet] 2014 [acesso em 2017 jul 1317]; 18(2). Disponível em: http://dx.doi. org/10.5935/1414-8145.20140045.

2. Torriani MS, Santos L dos, Echer IC, Barros E. Medicamentos de A a Z 2016/2018: enfermagem. Porto Alegre: Artmed; 2016.

3. Boullata JI, Carrera AL, Harvey L, Escuro AA, Hudson L, Mays A, et al. ASPEN Safe Practices for Enteral NutritionTherapy.Journal of Parenteral and Enteral Nutrition.[Internet] 2016 [acesso em 2017 jul 13]; 41(1). Disponível: http://dx.doi.org/ 10.1177/0148607116673053.

4. Brasil. Ministério da Saúde. Sistema Nacional de Auditoria / MS e Anvisa anunciam ações para segurança do paciente. [Internet] Brasília: Ministério da Saúde; 2013. [acesso em 2016 nov 11]. Disponível em:http://sna.saude. gov.br/noticias.cfm?id=5012.

5. Gimenes FRE; Reis RK. Manuseio de sonda enteral: uma revisão integrativa da literatura. Prática Hospitalar. [Internet] 2015 [acesso em 2016 nov11]; (97). Disponível em: https://www.researchgate.net/publication/279884916_ Manuseio_de_sonda_enteral_uma_revisao_integrativa_da_literatura.

6. White R, Bradnam V. Handbook of Drug Administratrion via Enteral Feeding Tubes. London: Pharmaceutical Press; 2011.

7. Moriel P, Shoji P, Bortoletto TC, Mazzola PG. Uso offlabel de medicamentos através de sondas: divergência entre informações. Rev Bras Farm Hosp Serv Saúde. [Internet] 2012 [acesso em 2016 nov 11]; 3(2). Disponível em: http://www.sbrafh.org.br/rbfhss/public/artigos/2012030204BR.pdf.

8. Hoefler R, Vidal JS. Administração de medicamentos por sonda. Boletim farmacoterapêutica. [Internet] Brasília: Conselho Federal de Farmácia; 2009 [acesso em 11 nov 2015]. Disponível em: http://www.cff.org.br/sistemas/ geral/revista/pdf/122/063a068_farmacoterapeutica.pdf.

9. Lisboa CD, da Silva LD, de Matos GC. Investigação da técnica de preparo de medicamentos para administração por cateteres pela enfermagem na terapia intensiva. Rev. esc. enferm. USP. [Internet] 2013 [acesso em 2016 nov 11]; 47(1). Disponível em: http://dx.doi.org/10.1590/S0080-62342013000100007.

10. Martins MR, Soares AQ, Modesto ACF, Carvalho RF, de Melo VV, Duarte IP. Análise de medicamentos administrados por sonda em unidades de terapia intensiva em hospital de ensino. Rev. Eletr. Enf. [Internet] 2013 [acesso em 2016 nov 11]; 15(1). Disponível em: https://doi.org/10.5216/ree.v15i1.15848.

11. Basso AP, Pinheiro MS. Avaliação dos medicamentos prescritos para pacientes submetidos à terapia nutricional enteral no CTI. Rev. Bras. Farm. Hosp. Serv Saúde.[Internet] 2014 [acesso em 2016 nov 11]; 5(1). Disponível em: http://www.sbrafh.org.br/rbfhss/public/artigos/2014050102000471BR.pdf.

12. Rodrigues JB, Martins FJ, Raposo NRB, Chicourel EL. Perfil de utilização de medicamentos por sonda enteral em pacientes de um hospital universitário. Rev. Bras. Farm. Hosp. Serv Saúde.[Internet] 2014 [acesso em 2016 nov 11]; 5(3). Disponível em: http://www.sbrafh.org.br/rbfhss/public/artigos/2014050305000592BR.pdf.

13. Heldt T, Loss SH. Interação fármaco-nutriente em unidade de terapia intensiva: revisão da literatura e recomendações atuais. RevBras Ter Intensiva. [Internet] 2013 [acesso em 2016 nov 11]; 25(2). Disponível em: http://dx.doi.org/10.5935/0103-507X.20130028. 
14. Nascimento MMG do, Ribeiro AQ. Compilação de base de dados com recomendações para administração de medicamentos via sonda enteral. Rev. Bras. Farm. Hosp. Serv Saúde. [Internet] 2010 [acesso em 2016 nov 11]; 1(1). Disponível em: http://www.sbrafh.org.br/rbfhss/public/artigos/RBFHSS_01_art04.pdf.

15. Reis VGO, Candido MF, de Jesús RP, Mendes-Netto RS. Perfil de utilización de medicamentos administrados por sonda enteral em el hospital universitário. Ver ChilNutr. [Internet] 2010 [acesso em 2016 nov 11]; 37(3). Disponível em: http://dx.doi.org/10.4067/S0717-75182010000300004.

16. Hulley SB, Cummings SR, Browner WS, Grady DG. Delineando a pesquisa clínica: uma abordagem epidemiológica. Porto Alegre: Artmed; 2008.

17. Universidade Federal do Rio Grande. Padronização de medicamentos. Gráfica da FURG, 2014.

18. Fontana RM. Assistência Farmacêutica na administração de fármacos via sonda enteral: um estudo personalizado. Revista Especialize On-line IPOG. [Internet] 2015 [acesso em 2016 nov 11]; 10(1). Disponível em: https://www.ipog.edu.br/revista-especialize-online/edicao-n9-2015/assistencia-farmaceutica-na-administracaode-farmacos-via-sonda-enteral-um-estudo-personalizado/.

19. Brasil. Ministério da Saúde. Secretaria de Ciência, Tecnologia e Insumos Estratégicos. Departamento de Assistência Farmacêutica e Insumos Estratégicos. Formulário terapêutico nacional 2010: Rename 2010. 2. ed. [Internet]Brasília: Ministério da Saúde; 2010 [acesso em 2016 nov 15]. Disponível em: http://bvsms.saude.gov.br/ bvs/publicacoes/formulario_terapeutico_nacional_2010.pdf.

20. Agência Nacional de Vigilância Sanitária (ANVISA). Bulário eletrônico. [Internet] Brasília: Ministério da Saúde; 2016 [acesso em 2016 out 02]. Disponível em: http://www.anvisa.gov.br/fila_bula/.

21. Silva MFB da. Perfil de medicamentos orais usados em uma unidade hospitalar de doenças infecciosas Elaboração de um manual de preparo e administração por cateteres enterais [Dissertação]. Rio de Janeiro (RJ): Instituto de Pesquisa Clínica Evandro Chagas; 2013.

22. Lima G de, Negrini NMM. Assistência farmacêutica na administração de medicamentos via sonda: escolha da forma farmacêutica adequada. Einstein. [Internet] 2009 [acesso em 2016 nov 2016]; 7(1). Disponível em: http:// apps.einstein.br/revista/arquivos/PDF/1035-einsteinv7n1p9_17.pdf.

23. Malagoli BG, Viel CB, Silva EF, Emery IC, Costa JM, Almeida KCA, et al. Manual para Melhoria das Práticas Assistenciais em Farmácia Hospitalar. Belo Horizonte: UFMG; 2009.

24. Hospital Sírio Libanês. Guia Farmacêutico 2014 / 2015 [Internet]. São Paulo: Hospital Sírio Libanês; 2015 [acesso em 2016 nov 11]. Disponível em: https://proqualis.net/sites/proqualis.net/files/Anexo2-\%20Guia\%20 Farmac\%C3\%AAutico\%202014.pdf.

25. Ferreira Neto CJB, Plodek CK, Soares FK, Andrade RA de, Teleginski F, Rocha MD. Pharmaceutical interventions in medications prescribed for administration via enteral tubes in a teaching hospital. Rev. Latino-Am. Enfermagem. [Internet] 2016 [acesso em 2016 nov 11]; 24. Disponível em: http://dx.doi.org/10.1590/1518-8345.0619.2696. 